\title{
Modeling radiation induced segregation in Iron-Chromium alloys
}

\author{
Oriane Senninger ${ }^{\mathrm{a}, *}$, Frédéric Soisson ${ }^{\mathrm{a}}$, Enrique Martínez ${ }^{\mathrm{b}}$, Maylise Nastar $^{\mathrm{a}}$, \\ Chu-Chun $\mathrm{Fu}^{\mathrm{a}}$, Yves Bréchet ${ }^{\mathrm{c}}$ \\ ${ }^{a}$ CEA, DEN, Service de Recherches de Métallurgie Physique, F-91191 Gif-sur-Yvette, \\ France \\ ${ }^{b}$ Los Alamos National Laboratory, Los Alamos, NM 87545, USA \\ ${ }^{c}$ SIMAP, INP Grenoble, CNRS UJF, Saint Martin d'Hères Cedex, France
}

\begin{abstract}
Radiation induced segregation in ferritic Fe-Cr alloys is studied by Atomistic Kinetic Monte Carlo simulations that include diffusion of chemical species by vacancy and interstitial migration, recombination, and elimination at sinks. The parameters of the diffusion model are fitted to DFT calculations. Transport coefficients that control the coupling between diffusion of defects and chemical species are measured in dilute and concentrated alloys. Radiation induced segregation near grain boundaries is directly simulated with this model. We find that the diffusion of vacancies toward sinks leads to a $\mathrm{Cr}$ depletion. Meanwhile, the diffusion of self-interstitials causes an enrichment of $\mathrm{Cr}$ in the vicinity of sinks. For concentrations lower than $15 \% \mathrm{Cr}$, we predict that sinks will be enriched with $\mathrm{Cr}$ for temperatures lower than a threshold. When the temperature is above this threshold value, the sinks will be depleted in Cr. These results are compared to previous experimental studies and models. Cases of radiation induced precipitation and radiation accelerated precipitation are considered.

Keywords: Fe-Cr alloys; Monte Carlo simulation; Diffusion; Irradiation effect; Segregation
\end{abstract}

\footnotetext{
* Corresponding author

Email address: oriane.senninger@northwestern.edu (Oriane Senninger )
}

Preprint submitted to Acta Materialia

September 25, 2015

(C) 2015. This manuscript version is made available under the Elsevier user license http://www.elsevier.com/open-access/userlicense/1.0/ 


\section{Introduction}

Ferritic Fe-Cr steels are good candidates as structural materials for the next generation of nuclear power plants (generation IV and fusion reactors) [1, 2. The addition of $\mathrm{Cr}$ prevents corrosion and the $\mathrm{Fe}-\mathrm{Cr}$ ferritic form is weakly 5 sensitive to the swelling phenomenon. However, the irradiation flux induces an increase of point defect concentrations - vacancies and self-interstitial atoms (I) - that migrate toward sinks where they are eliminated. These defect fluxes may induce a variation in the alloy composition in the vicinity of sinks called Radiation Induced Segregation (RIS) [3]. If this causes a Cr depletion, the alloy can become sensitive to corrosion. On the other hand, if this leads to a $\mathrm{Cr}$ enrichment, the local concentration can reach the solubility limit, causing Radiation Induced Precipitation (RIP), which could lead to embrittlement. RIS has been extensively studied in austenitic steels where $\mathrm{Cr}$ depletion and $\mathrm{Ni}$ enrichment are frequently observed at sinks. These tendencies are relatively well-understood, even if the interstitial contribution is still under discussion [3]. In ferritic steels, the situation is far from being clear: approximately 15 experiments have been reviewed by $\mathrm{Lu}$ et al. [4] in very different materials and conditions, showing both depletions and enrichments in the vicinity of sinks. However, it is difficult to draw clear conclusions on how irradiation conditions and materials properties control the tendency towards enrichment or depletion. A systematic study, focusing on industrial steels with $\mathrm{Cr}$ content between 8 and $12 \%$ has been performed by Was et al. 5. They observed that for given irradiation conditions, sinks tend to be enriched in $\mathrm{Cr}$ at low temperature and to be depleted in $\mathrm{Cr}$ at high temperature. Moreover, the $\mathrm{Cr}$ enrichment tends to decrease with the alloy $\mathrm{Cr}$ content. These tendencies are reproduced with the inverse Kirkendall model of Wharry et al. [6] and are attributed to a positive coupling of $\mathrm{Cr}$ with self-interstitials (dominant at low temperatures) and a negative coupling with vacancies (dominant at high temperatures). Other factors, such as grain boundary misorientation [7, interactions with $\mathrm{C}$ or other impurities [7, 8] may influence the segregation of $\mathrm{Cr}$ at sinks. 
In the present study, we limit ourselves to the Fe-Cr binary system in order to understand basic mechanisms controlling RIS at the atomic scale. Thermodynamics of Irreversible Processes (TIP) shows that a complete description of RIS requires a full determination of the Onsager coefficients $L_{i j}$, or alternatively, 35 of partial diffusion coefficients $d_{i j}$ [3]. Such a consistent description remains to be established for concentrated Fe-Cr alloys. Existing models are based on Density Functional Theory (DFT) calculations and multi-frequency models that are only valid in the dilute limit [9, 10, or have been extended to concentrated alloys using additional approximations [9, 5, 6. Others use Molecular Dynam-

40 ics (MD) simulations that rely on empirical potentials and only determine the self-interstitial contribution [11, 12].

We present here a multiscale approach of RIS, starting from DFT calculations of the point defect migration energies. A diffusion model describing the variation of point defect properties with local composition is developed. Atom-

45 istic Kinetic Monte Carlo (AKMC) simulations are performed in order to (i) determine the $L_{i j}$ coefficients and predict the RIS tendencies; and (ii) model the evolution of point defect and $\mathrm{Cr}$ concentration profiles under irradiation in the vicinity of a grain boundary (GB). AKMC simulations include the effect of non-homogeneous concentration fields and account for the stochastic nature 50 of point defect migration. It also enables the simulation of RIP, when the RIS leads to a strong enrichment of Cr near GBs and therefore to nucleation of $\alpha$ ' precipitates.

In section II we present a reminder of RIS principles in the framework of TIP. In section III, the diffusion model and the AKMC method are introduced. ${ }_{55}$ The fitting of parameters to DFT calculations is detailed and relevant point defects properties are discussed. In section IV, the point defect tracer diffusion coefficients and Onsager coefficients are analyzed. The effect of composition and temperature on these Onsager coefficients is studied. Simulations of RIS and RIP near grain boundaries are also shown. We discuss these results by compar${ }_{60}$ ing them to previous models and experiments. We conclude by commenting on the limitations of our model, possible improvements and perspectives. 


\section{Thermodynamics of Irreversible Processes and Radiation Induced Segregation}

RIS is due to fluxes of vacancies $\left(J_{V}\right)$ and self-interstitials $\left(J_{I}\right)$ towards point defect sinks that, in an A-B alloy, induce fluxes of chemical species $\left(J_{A}\right.$ and $\left.J_{B}\right)$. TIP describes the fluxes $J$ as linear combinations of thermodynamic driving forces, i.e. chemical potential gradients [3]:

$$
J_{\alpha}=-\Sigma_{\beta} L_{\alpha \beta} X_{\beta}
$$

with $X_{\beta}=\nabla \mu_{\beta} /\left(k_{B} T\right) . \quad L_{\alpha \beta}$ are the Onsager coefficients, $\mu_{\beta}$ the chemical potentials, $k_{B}$ the Boltzmann constant and $T$ the temperature.

In steady state, under the conditions where there is no precipitation and $L_{\alpha \beta}$ coefficients are independent of the local composition, the gradient of B and $\mathrm{V}$ near a sink are related by:

$$
\nabla C_{B}=-\frac{L_{A I} L_{A V}}{C_{V}\left(L_{A I} D_{B}+L_{B I} D_{A}\right)}\left(\frac{L_{B V}}{L_{A V}}-\frac{L_{B I}}{L_{A I}}\right) \nabla C_{V}
$$

where $D_{A}$ and $D_{B}$ are intrinsic diffusion coefficients. This profile may also be expressed in term of partial diffusion coefficients: $d_{\alpha V}=L_{\alpha V} / C_{\alpha} C_{V}$ and ${ }_{75} d_{\alpha I}=L_{\alpha I} / C_{\alpha} C_{I}[13,14$.

The sign of the $L_{\alpha \beta}$ coefficients corresponds to the direction of couplings. For example, in the A-B alloy if $L_{B V}>0$ fluxes of $\mathrm{B}$ and $\mathrm{V}$ are in the same direction and if $L_{B V}<0$ they are in opposite directions. In simple cases, general rules of concentration variations can be derived from point defect diffusion coefficients. RIS is thus often analyzed in terms of Inverse Kirkendall effect: for the vacancies, if $\mathrm{B}$ is the fast diffusing species (ie $D_{B}>D_{A}$ ), a depletion of $\mathrm{B}$ at sinks is expected (note that it is only true when $L_{B V}<0$ ); for interstitials, if $\mathrm{B}$ is the fast diffusion species, an enrichment of $\mathrm{B}$ is expected ( $L_{B I}$ is always positive) [3]. On the other hand, in the case of significant attraction between solute atoms and vacancies, $L_{B V}$ may be positive, leading to solute atoms dragged by vacancies and a solute enrichment at sinks (this is called the drag effect). Beyond such 
interpretations, one must know the full set of $L_{i j}$ coefficients in Eq. 2 and how they depend on temperature and concentration in order to determine the RIS profiles.

The $L_{i j}$ coefficients are very difficult to extract from diffusion experiments but they can be in principle easily deduced from atomic scale simulations. This requires: (i) a good description of jump frequencies and their variation with respect to the local environment and (ii) a good method to compute the $L_{i j}$ coefficients from the jump frequencies. For the second point, two strategies are possible:

1. A numerical determination of the $L_{i j}$ (or $d_{i j}$ ) coefficients by MD or AKMC simulations. MD simulations with an empirical potential have been used by Terentyev, Pechenkin et al. [11, 12] for the coefficients of self-interstitials. Here we use AKMC simulations to directly measure the $L_{i j}$ coefficients of both vacancies and self-interstitials.

2. An analytical calculation of the $L_{i j}$ coefficients (or alternatively, of the partial diffusion coeffcients) from point defects jump frequencies, using multi-frequency diffusion models valid for dilute systems. This approach has been applied to Fe-Cr alloys by Choudhury et al. [9] and by Was, Wharry et al. [5, 6]. Those diffusion models were limited to short-range interacting alloys and the contribution to the $L_{i j}$ coefficients of some vacancy jumps beyond the first nearest neighbor sites of the solute atom had to be neglected. Recently, long-range interaction models of the $L_{i j}$ coefficients have been developed [15, 16]). In concentrated alloys, due to the high complexity, approximate mean field methods are used to determine the $L_{i j}$ coefficients $[3$.

\section{Diffusion model and AKMC simulations}

\subsection{Diffusion model and effective pair interactions}

The calculation of point defects jump frequencies is based on a rigid lattice model using effective pair interactions hereafter referred to as the "AKMC 
model". We start from a previous version [17] that only includes vacancy diffusion. It assumes that the free enthalpy of a given atomic configuration can be written as a sum of effective pair interactions on a rigid bcc lattice.

Such "broken bond" models with constant pair interactions are widely used in AKMC simulations to model diffusive phase transformations in alloys [18, 19, 20, 21. However, Fe-Cr alloys have a special thermodynamic behaviour, with strong vibrational and magnetic contributions. The latter are especially important: they lead to a change in the sign of the alloy mixing energy around 10 at.\% Cr. In ref [17], magnetic and vibrational contributions are taken into account by introducing a temperature dependence on all interactions and by making Fe-Cr interactions on lattice sites dependent on local concentration (see Refs. [22, 17]). This model generates solubility limits in good agreement with recently updated Fe-Cr phase diagrams [23, 24], showing an asymmetrical miscibility gap (see fig. 1 in Ref [17]). It gives diffusion coefficients and kinetics of precipitation in good agreement with experimental data, in the range of temperatures and compositions considered here (300-950 K and up to $20 \%$ of $\mathrm{Cr}$ ). 1

In the present study, the diffusion of self-interstitials is modeled in a similar manner. As we will see in the next section, dumbbells with a $\langle 110\rangle$ orientation have the most stable interstitial configuration in Fe and dilute Fe-Cr alloys. We therefore only consider this configuration. The migration occurs through the Johnson mechanism [25]: a jump of the dumbbell towards a nearest-neighbor atom, with a rotation of $60^{\circ}$. Two types of nearest neighbor sites can be distinguished : four sites in tension (T) and four sites in compression (C) [26]. Self-interstitial atoms can only jump to compression sites.

The jump frequency for an exchange between an atom $A(=\mathrm{Fe}, \mathrm{Cr})$ and a self-interstitial $I$ is given by $\Gamma_{A I}=\nu \exp \left(-\Delta G_{A I}^{m i g} / k_{B} T\right)$, where $\nu=10^{13} \mathrm{~s}^{-1}$ is

\footnotetext{
${ }^{1}$ In Fe-Cr solid solutions, the ferro-to-paramagnetic transition leads to an increase of the diffusion coefficients that can be modeled by a correction of the vacancy migration barriers [17. It is shown to significantly accelerate the precipitation kinetics in alloys with high $\mathrm{Cr}$ contents (above typically 25\%). As we limit our study to concentrations lower than $\sim 15 \%$, such effects are not included in the present model.
} 
the Debye frequency and $\Delta G_{A I}^{m i g}$ the migration barrier :

$$
\Delta G_{A I}^{m i g}=\Sigma_{j} \tilde{g}_{A I j}-\Sigma_{k, n} g_{A k}^{(n)}-\Sigma_{l} g_{I l}-g_{I i}-\Sigma_{p q, n} \Delta g_{p q}^{(n)}
$$

where the first term on the right hand side corresponds to interactions created The results are very similar to those of Choudhury et al. 9] and Messina et al. [16]. These studies show a weak $\mathrm{Cr}-\mathrm{V}$ interaction and a low migration barrier for the $\mathrm{Cr}-\mathrm{V}$ exchange (corresponding to the $W_{2}$ frequency using the 
vacancy jumps. This results in a rapid diffusion of $\mathrm{Cr}$ in $\mathrm{Fe}$ (tracer diffusion coefficients $\left.D_{C r^{*}}>D_{F e^{*}}\right)[17$.

The pair interactions of the AKMC model have been fitted to some of these DFT results. In most cases, migration barriers and binding enthalpies generated 175 by this model are in good agreement with the DFT calculations. The migration barriers of jump frequencies $W_{3}$ and $W_{4}$ are slightly higher than the DFT values. Meanwhile, the binding energy between $\mathrm{Cr}$ and the vacancy is slightly negative (almost zero) in the AKMC model, while it is slightly positive in the DFT calculations. $W_{3}$ and $W_{4}$ jump frequencies may affect the rotation of the vacancy around solute atoms and therefore the drag effect [15, 10. In spite of these differences, the model gives tracer and interdiffusion coefficients in good agreement with experimental data in ferromagnetic alloys [17, 28].

\subsubsection{Self-interstitial atoms properties}

Self-interstitial properties have been studied by Olsson et al. [29] in dilute FeFe-Cr alloys using the 2BM empirical potential.

We present here a DFT study of self-interstitial properties (formation, migration and binding energies) in pure metals, dilute and more concentrated $\mathrm{Fe}-\mathrm{Cr}$ alloys, with the objective of fitting the corresponding AKMC parameters.

DFT calculations were performed using the PWSCF code [30]. We performed spin-polarized calculations within the generalized gradient approximation (GGA). Accurate projector augmented wave (PAW) potentials were used, where the $3 \mathrm{~d}$ and the $4 \mathrm{~s}$ electrons were considered as valence electrons. The kinetic energy cutoff chosen for the plane-wave basis set was $544 \mathrm{eV}$. All the calculations were carried out with 128-atom supercells, using a $(3 \times 3 \times 3) k$ point grid and the Methfessel-Paxton broadening scheme with a $0.3 \mathrm{eV}$ width. To calculate the energetics of interstitial atoms, we have adopted a constant volume-per-atom approach. For instance, the lattice parameter of a cubic supercell containing $N$ Fe lattice atoms and one Fe interstitial was rescaled in 
Pure metals.

Table 1: Self-interstitial atoms formation energies (in eV) in pure Cr, computed in DFT-PAW calculations

\begin{tabular}{|l|l|l|}
\hline configuration & Cr-NM & Cr-AFM \\
\hline 100 & 6.63 & 6.84 \\
\hline 110 & 5.58 & 6.08 \\
\hline 111 & 5.64 & 6.20 \\
\hline Octahedral & 6.76 & 7.04 \\
\hline Tetrahedral & 6.31 & 6.60 \\
\hline
\end{tabular}

In pure iron, our DFT calculations give a formation enthalpy of $3.96 \mathrm{eV}$ for the $\langle 110\rangle$ dumbbell, the most stable orientation in pure iron (in agreement with previous studies [31, 25]). The $\langle 111\rangle$ dumbbell has a formation enthalpy of $4.74 \mathrm{eV}$.

Formation energies of different self-interstitial configurations in pure $\mathrm{Cr}$, with a non-magnetic (NM) or anti-ferromagnetic (AFM) state, are reported in Table 1. Olsson et al. 29 have obtained values slightly smaller but with almost the same relative stability between the different configurations. The magnetic state 220

volume in a defect-free bcc lattice. The atomic positions were fully relaxed with

The binding energy of a configuration containing $n$ point defects noted $d_{i}$ is given by:

$$
E_{b}(\text { config })=\sum_{i=1}^{n} E\left(d_{i}\right)-E(\text { config })-(n-1) E_{r e f}
$$

where $E_{r e f}$ is the total energy of a supercell of pure Fe, $E\left(d_{i}\right)$ is the total energy of a supercell containing the single defect $d_{i}$ and $E$ (config) is the total energy of the supercell containing all the defects $\left(d_{i}\right)$. A positive binding energy refers

The migration barriers have been determined using the drag method as in our previous work [17].

has a very limited effect on the relative stability of the different configurations. 
The $\langle 110\rangle$ configuration is the most stable one in each case, but the formation energy of $\langle 111\rangle$ is very close. The formation energies of the $\langle 100\rangle$ dumbbell, octahedral and the tetrahedral configurations are significantly higher.

Binding energies in $\mathrm{Fe}-\mathrm{Cr}$ alloys.

Binding energies have been calculated for 52 configurations involving a $\langle 110\rangle$ dumbbell in $\mathrm{Fe}$, with up to four $\mathrm{Cr}$ atoms in first and second nearest neighbor positions. Table 2 presents a selection of binding energies computed in DFT and with our AKMC model. Fig. 1 summarizes the comparison between the DFT calculations and the AKMC model for all configurations. A few binding energies between Fe atoms and $\langle 110\rangle$ dumbbells in $\mathrm{Cr}$, and between $\mathrm{Cr}$ and $\langle 111\rangle$ dumbbells in Fe are also given on Table. 2.

In the 52 configurations of Fig. 1 the $\langle 110\rangle$ dumbbell is more stable than the $\langle 111\rangle$, except for two cases. In these two cases, the $\langle 111\rangle$ configuration is an energy slightly lower than the $\langle 110\rangle$ configuration, but is still negative with a strongly negative energies (respectively -0.63 and $-0.80 \mathrm{eV}$ ) and therefore have negligible probabilities of occurrence.

The mixed interstitial (line 1 in Table 2) is stable in pure Fe, with a positive binding energy $(+0.022 \mathrm{eV})$. The interactions between a Fe-Fe $\langle 110\rangle$ dumbbell and a $\mathrm{Cr}$ atom on tension site (line 2) are repulsive $(-0.038 \mathrm{eV})$. The ones with a $\mathrm{Cr}$ atom on a compression site (line 3$)$ are attractive $(+0.016 \mathrm{eV})$.

Cr-Cr dumbbells are strongly repulsive in pure iron (line 11, $-0.554 \mathrm{eV}$ ) and more generally in all the dilute Fe-Cr configurations that we have considered. The binding energy of a mixed interstitial with a $\mathrm{Cr}$ atom on a tension site is negative (line 7) whereas the one with a $\mathrm{Cr}$ atom on a compression site 245 depends on the position of the $\mathrm{Cr}$ atom within the dumbbell (lines 8 and 9). Configurations with more $\mathrm{Cr}$ atoms (e.g. lines 10,11 and 12) usually have very negative binding energies. This results from the magnetic frustration occurring between $\mathrm{Cr}$ neighbors in Fe. These frustrations explain the ordering tendency of dilute $\mathrm{Fe}-\mathrm{Cr}$ alloys [32]. However, some configurations with two or more $\mathrm{Cr}$ atoms have positive binding energies (especially with $\mathrm{Cr}$ on compression sites, 
e.g. on lines 4, 6, 9) and may tend to decrease the mobility of dumbbells.

In pure $\mathrm{Cr}$, the mixed and Fe-Fe $\langle 110\rangle$ dumbbells (lines 13 and 14) are very attractive, with high binding energies respectively close to 1 and $2 \mathrm{eV}$.

In general, our results are similar to the previous DFT results of Olsson et al.

[29]. The results obtained by Terentyev et al. [11] with the 2BM potential tend to overestimate the attraction between $\langle 110\rangle$ dumbbells and $\mathrm{Cr}$ atoms compared to DFT calculations. As mentioned in [11, the binding energies between $\langle 111\rangle$ dumbbells and $\mathrm{Cr}$ atoms are positive (lines 15 and 16), but they are lower than the difference between the formation energies of $\langle 111\rangle$ and $\langle 110\rangle$ dumbbells in pure Fe (approximately $0.8 \mathrm{eV}$ ): these $\langle 111\rangle$ configurations are therefore not probable and should not be able to trap the self-interstitials.

The binding energies of the AKMC model are usually in good agreement with the corresponding DFT values (Fig. 1), especially for configurations involving only 1 or $2 \mathrm{Cr}$ atoms. In the case of configurations including 3 and $4 \mathrm{Cr}$ atoms, the AKMC model reproduces the general trend, i.e. strongly negative binding energies.

\subsubsection{Migration energies in Fe-Cr alloys}

DFT calculations of migration barriers between configurations involving one $\langle 110\rangle$ dumbbell and between 1 and $4 \mathrm{Cr}$ atoms are given in Fig. 2 ,

The present calculations give a migration energy of $0.34 \mathrm{eV}$ for the $\langle 110\rangle$ dumbbell in pure iron, in agreement with previous studies [25, 26, 9]. In alloys, our DFT study shows that this mechanism is associated with the lowest migration energy of the $\langle 110\rangle$ dumbbell. The mixed interstitial has a migration barrier significantly lower than the Fe-Fe dumbbell in pure iron: 0.23 instead of $0.34 \mathrm{eV}$.

275 The migration barriers of the Cr-Cr dumbbells are even lower $(\sim 0.05 \mathrm{eV})$ but configurations involved in these processes are very repulsive $\left(E_{b} \ll 0\right)$.

In configurations with one $\mathrm{Cr}$ atom, the migration barriers are close to those of Choudhury et al. [9] and Olsson et al. [26]. Choudhury et al. [9] have also computed migration barriers for self-interstitials jumps without rotation. Their migration barriers are systematically higher when compared to the Johnson 
mechanism, which further validates our choice of migration mechanism.

For migration barriers with 1 or $2 \mathrm{Cr}$ atoms, the AKMC model is in good agreement with the DFT calculations (Fig.2). For higher Cr concentrations, the discrepancy can be as high as $0.4 \mathrm{eV}$. However, they correspond to configurations with negative binding energies, i.e. low probabilities. A better agreement could be obtained by introducing pair interactions between dumbbells and atoms at positions further than first nearest neighbors and a composition dependence to the saddle-point pair interactions. However, as this would slow down the AKMC code, we prefer to leave this improvement for a future study.

Finally, the attempt frequency of vacancy jumps is assumed to be $\nu_{0}=$ $10^{13} \mathrm{~s}^{-1}$ and the migration barriers include a migration entropy of $2.1 k_{B}$ [17. The migration entropy of interstitials has never been experimentally measured. For the sake of simplicity, it has been chosen here to take the same value as the vacancies.

To summarize, key self-interstitials properties revealed by DFT calculations and reproduced by the AKMC model are: the moderate attraction between interstitials and $\mathrm{Cr}$ atoms in dilute $\mathrm{Fe}$, the stability of mixed interstitials in dilute $\mathrm{Fe}$ and the rapid migration of the mixed interstitial.

\subsection{AKMC simulations}

Two kinds of AKMC simulations have been performed: measurements of transport coefficients and simulations of RIS near a grain boundary.

For the first ones, one point defect (V or I) is introduced in a simulation box with $N=4096$ bcc sites, periodic boundary conditions and up to $15 \%$ of $\mathrm{Cr}$. The jump frequencies are computed with our diffusion model and a residence time algorithm (RTA) is used to choose the jumps and to increment the time [33. Tracer diffusion coefficients of point defects $d=I, V$ and $L_{i j}$ coefficients are computed with generalized Einstein's relations [34]:

$$
D_{d}=\frac{\left\langle\triangle r_{d}^{2}\right\rangle}{6 t}
$$

and 


$$
L_{i j}=\frac{1}{6 V_{t o t} k_{B} T} \frac{\left\langle\triangle R_{i} \triangle R_{j}\right\rangle}{t},
$$

where $\triangle r_{d}$ is the average displacement of defect $d$ during time $t . \triangle R_{i}$ is the total

displacement of species or defect $i$ during time $t$ and $V_{\text {tot }}$ is the total volume of the system. To obtain sufficient statistics, average values are calculated on $10^{5}$ series of $10^{5}$ Monte Carlo steps. Measurements are performed in homogeneous solid solutions, i.e. outside the miscibility gap or slightly inside - but in the latter case checking that $\alpha^{\prime}$ precipitation does not occur during the measurements.

Simulations of RIS at grain boundaries are performed in samples with $N=$ $512 \times 64 \times 64$ bcc sites, with a GB located in the middle of the long dimension. These simulations take into account the following events [35, 36]:

- the creation of Frenkel pairs $(\mathrm{I}-\mathrm{V})$ with a production rate $K_{0}$. I-V pairs are created randomly within the simulation box, along sequences of 10 displacements in $\langle 111\rangle$ directions. This mechanism corresponds to electron rather than neutron or heavy ion irradiation.

- the migration of I and V, according to the diffusion model,

- the recombination of I and $\mathrm{V}$ pairs below a recombination radius $R_{c}$ of 4 nearest neighbors.

- the annihilation of I and V when they jump on a GB. Within our rigid approach, the GB is simply modeled as a planar perfect sink.

\section{Results}

\subsection{Point defect tracer diffusion coefficients}

We present first diffusion coefficients of point defects which determine the steady state point defect concentration under irradiation. The tracer diffusion coefficients of one vacancy and one interstitial in alloys at nominal concentrations up to $15 \% \mathrm{Cr}$ and at temperatures between $300 \mathrm{~K}$ and $1500 \mathrm{~K}$ are presented in Figures 3 . The vacancy diffusion coefficients slightly increase with the $\mathrm{Cr}$ concentration, due to the low $\mathrm{Cr}-\mathrm{V}$ exchange barrier in Fe. 

at $300 \mathrm{~K}$ ) than the vacancy diffusion coefficients. It has to be noted that a change of interstitial migration entropy in the energetic model would change the I diffusion coefficients. The effect of $\mathrm{Cr}$ concentration on the I diffusion coefficient depends on temperature. At temperatures lower than the threshold value $T_{s} \sim 600 \mathrm{~K}$, the addition of $\mathrm{Cr}$ induces an acceleration of self-interstitial diffusion. At $T>T_{s}$ the inverse tendency is observed.

The increase of the interstitial diffusion coefficient with concentration at low temperature is due to the stability and the low migration barrier of the mixed dumbbell. The influence of $\mathrm{Cr}$ concentration on self-interstitial diffusion at high temperature can be explained by the dissociation of the mixed interstitials and the trapping of I on $\mathrm{Cr}$ atoms. These results are in qualitative agreement with the MD simulations of Terentyev, Pechenkin et al., using the 2BM empirical potential [11, 12]. A fit of the self-interstitial diffusion coefficients by an Arrhenius law: $D_{I}=D_{I}^{0} \exp \left(-\Delta H_{I}^{m i g} /\left(k_{b} T\right)\right.$ gives $H_{I}^{m i g}=0.34,0.29,0.27,0.25 \mathrm{eV}$ for $0.25,5,10$ and $15 \% \mathrm{Cr}$ respectively, close to the values of Terentyev et al. (Table 2 in ref. [11]). However, in their MD simulations, the threshold temperature is lower $\left(T_{s} \sim 400 \mathrm{~K}\right)$. This may be related to the overestimation of the $\mathrm{I}-\mathrm{Cr}$ attraction by the $2 \mathrm{BM}$ potential, as the trapping effect becomes dominant at lower temperatures.

\subsection{Onsager coefficients}

We have measured that for concentrations ranging from 0.25 to $15 \% \mathrm{Cr}$ and temperatures between 300 and $1500 \mathrm{~K}$, the $L_{F e I}$ and $L_{C r I}$ coefficients are positive and the $L_{\mathrm{FeV}}$ and $L_{\mathrm{CrV}}$ coefficients are negative. Fe and $\mathrm{Cr}$ atoms diffuse thus in the opposite direction than vacancies and in the same direction as interstitials.

In the dilute limit, one observes the theoretical result $L_{F e V} / L_{F e F e}=-1$. The $L_{C r V} / L_{C r C r}$ ratio is close to -2 which indicates a strong negative coupling between vacancies and $\mathrm{Cr}$ atoms displacements, as could be expected from the small migration barrier $\Delta H_{2}$ for the $\mathrm{Cr}-\mathrm{V}$ exchange in Fe and from the absence 

itation occurs rapidly below $600 \mathrm{~K}$, preventing measurement of the Onsager 
coefficients, but the threshold value can be roughly estimated to be $400-500 \mathrm{~K}$ by extrapolation). These two features are in qualitative agreement with the experimental observations and simulations of Wharry et al. [6] performed in alloys with concentrations between 8 and $12 \% \mathrm{Cr}$.

For self-interstitials, these results can be compared with the MD study by Pechenkin et al. 12, performed in a similar range of concentrations. They give a ratio of partial diffusion coefficients $d_{C r I} / d_{F e I}$ decreasing from $\sim 3.7$ to $\sim 2.2$ in Fe-5\%Cr between 600 and $1000 \mathrm{~K}$, and from $\sim 1.8$ to $\sim 1.2$ in Fe-10\%Cr. With $d_{C r I} / d_{F e I}=\left(C_{F e} L_{C r I}\right) /\left(C_{C r} L_{F e I}\right)$, our AKMC simulations give higher ratios and higher variations: from $\sim 8$ to $\sim 3$ in both alloys. Penchenckin et al. [12] have measured the tracer diffusion coefficients of Fe and $\mathrm{Cr}$ by self-interstitial diffusion in Fe-Cr alloys through MD simulations and have derived an estimation of the partial diffusion coefficients based on the Manning's approximation. It is thus difficult to determine if the discrepancy comes from this approximation or from differences in the self-interstitials jump frequencies. Nevertheless, the present study confirms the main conclusions of these approaches, without relying on similar approximations.

\subsection{Radiation induced segregation at grain boundaries}

AKMC simulations of RIS near point defect sinks have been performed to check the predictions deduced from the measurements of the $L_{i j}$ coefficients. Contrary to Eq. 2 they take into account the variation of transport coefficients with the solute concentration in the vicinity of the sink. Moreover, they can model precipitation as well as segregation phenomena. We first present steady state concentration profiles when no precipitation is observed and a kinetic study of RIS and then analysis of precipitation in undersaturated and oversaturated alloys. In these simulations, Cr segregation profiles across the grain boundary are characterized by the concentration on the sink plane $\left(C_{C r}^{G B}\right)$ and by the total quantity of $\mathrm{Cr}$ segregated $\left(S_{C r}^{t o t}=\int\left(\frac{C_{C r}}{C_{F e}}(x)-\frac{C_{C r}^{0}}{C_{F e}^{0}}\right) d x\right.$ where $x$ is the direction perpendicular to the sink and $C_{C r / F e}^{0}$ is the $\mathrm{Cr} / \mathrm{Fe}$ concentration in the bulk) [37. 
Simulations have been performed for alloy compositions from 1 to $15 \% \mathrm{Cr}$, at $650 \mathrm{~K}$ and $950 \mathrm{~K}$. Simulations at $650 \mathrm{~K}$ were performed with a flux of $10^{-6} \mathrm{dpa} . \mathrm{s}^{-1}$ and the one at $950 \mathrm{~K}$ with a flux of $10^{-3} \mathrm{dpa} . \mathrm{s}^{-1}$.

Point defects and $\mathrm{Cr}$ concentration profiles obtained in simulations at $10 \% \mathrm{Cr}$ and at the two temperatures are reported in Fig. 5. Steady state point defect concentrations far from sinks are given by:

$$
\begin{aligned}
C_{V} & =-\frac{K_{I}}{2 R}+\sqrt{\frac{K_{I}^{2}}{4 R^{2}}+\frac{K_{0} K_{I}}{R K_{V}}} \\
C_{I} & =-\frac{K_{V}}{2 R}+\sqrt{\frac{K_{V}^{2}}{4 R^{2}}+\frac{K_{0} K_{V}}{R K_{I}}}
\end{aligned}
$$

with $K_{I}=k_{I s} D_{I}, K_{V}=k_{V s} D_{V}$, where $k_{I s}$ and $k_{V s}$ terms are the sink strengths for point defects elimination [38], $R=4 \pi R_{c}\left(D_{I}+D_{V}\right) / \Omega$ with $R_{c}$ the critical distance of recombination, $K_{0}$ the point defect production rate and $\Omega$ the alloy atomic volume. These equations predict concentrations of vacancies and selfinterstitials respectively of $1.5 \times 10^{-11}$ and $2 \times 10^{-13}$ at $650 \mathrm{~K}$. At $950 \mathrm{~K}$, these concentrations change to $4.5 \times 10^{-10}$ and $4.5 \times 10^{-11}$. Our simulations show a fairly good agreement with these values. As interstitials diffuse faster than vacancies (see Fig. 3), they reach the GB more rapidly and their concentration in the bulk is lower (Fig. 5) : at steady state $C_{I} D_{I}=C_{V} D_{V}$.

At $950 \mathrm{~K}$ one observes a depletion of $\mathrm{Cr}$ near the GB (see Fig. 5 as an example) for all compositions. At $650 \mathrm{~K}$, the observed evolutions depend on the alloy concentration. At $1 \% \mathrm{Cr}$, we observe no evolution of the alloy composition at sinks. At 5 and $10 \% \mathrm{Cr}$, the $\mathrm{GB}$ tends to be enriched in $\mathrm{Cr}$ with a larger concentration increase for the alloy at $5 \% \mathrm{Cr}\left(C_{C r}^{G B}\right.$ goes from $5 \%$ to $\left.11 \%\right)$ than for the one at $10 \% \mathrm{Cr}\left(C_{C r}^{G B}\right.$ goes from $10 \%$ to $\left.13 \%\right)$. This RIS behavior is in agreement with the $L_{i j}$ measurements summarized in Fig. 4 .

The kinetic evolution of $\mathrm{Cr}$ segregation profile according to irradiation dose has been analyzed for the simulation at $10 \% \mathrm{Cr}$ and $650 \mathrm{~K}$ using a box of $256 \times 64 \times$ 64 bcc sites (see Fig. 6). We observe that the concentration at sinks reaches a 
steady state very fast and that the total quantity of $\mathrm{Cr}$ segregated evolves much slower (an exponential fit of these evolutions gives a relaxation dose of 0.023 dpa for $C_{C r}^{G B}$ and $0.107 \mathrm{dpa}$ for $\left.S_{C r}^{\text {tot }}\right)$. It has to be noted that the difference in kinetics between $C_{C r}^{G B}$ and $S_{C r}^{\text {tot }}$ observed in our simulations are in agreement with experimental observations in $\mathrm{Ni}-\mathrm{Cu}$ [39] and Ni-Si [40] alloys. Moreover, by multiplying and dividing the density of sinks by a factor 2 , we observe that $S_{C r}^{\text {tot }}$ decreases with the density of sinks but that $C_{C r}^{G B}$ does not evolve much with it. From our best knowledge, kinetic evolutions of concentration profiles at sinks have never been observed in the Fe-Cr alloy at such low doses. Wharry et al. 41] have nevertheless computed with their Inverse Kirkendall model a significant enrichment at sinks at low doses (lower than $10^{-3}$ dpa) and predict a steady state of $\mathrm{Cr}$ enrichment which is reached at 1 dpa or less. However, their measurements on GBs show that concentration profiles keep evolving in doses higher than $1 \mathrm{dpa}$ 41. This evolution is attributed to microstructural evolutions during irradiation. In particular, the flux of ions induces an evolution of the dislocation loop density which leads to a change of defect concentration and so to an evolution of RIS profiles.

At low temperatures, the Cr enrichment may be sufficient to reach the solubility limit, even if the alloy is undersaturated. In such cases one observes a local Radiation Induced Precipitation near the GB. An example is given in an alloy at $9 \% \mathrm{Cr}$ and at $563 \mathrm{~K}$ on Fig. 7 . In this case, Eqs. (7) and (8) give the concentrations of vacancies and self-interstitials respectively of $1.5 \times 10^{-10}$ and $4.5 \times 10^{-13}$ in agreement with the simulation.

The RIS phenomenon can also be associated with radiation accelerated precipitation. Indeed, at $15 \% \mathrm{Cr}$ and $650 \mathrm{~K}$, the analytic RIS model predicts a depletion of $\mathrm{Cr}$ at sinks (see Fig. 4). However, at this composition and temperature, the alloy is in the miscibility gap and decomposes into $\alpha$ and $\alpha^{\prime}$ phases. ${ }_{475}$ As shown in Fig. 8, we observe the formation of $\alpha^{\prime}$ precipitates and a small depletion of $\mathrm{Cr}$ in the vicinity of the sink. This decomposition is accelerated compared to thermal equilibrium as point defect concentrations are increased by irradiation in the bulk. In the GB vicinity, point defect concentrations de- 
crease (Fig. 8(a)) and the nucleation, growth and coarsening of $\alpha^{\prime}$ precipitates is much slower than in the bulk: one observes a precipitate free zone around a GB. Such precipitate free zones have been experimentally observed by 3D Atom Probe during the $\alpha^{\prime}$ precipitation near grain boundary in ferritic steels [42]. In Figs. 7 and 8 we observe some small peaks in self-interstitial concentration profiles. These peaks are due to trapping of self-interstitials in configurations highly concentrated in $\mathrm{Cr}$ and at $\alpha / \alpha^{\prime}$ interfaces.

\section{Conclusions}

In this study we have analyzed radiation-induced segregation (RIS) in the binary bcc Fe-Cr alloy with a new Atomistic Kinetic Monte Carlo model. The previous RIS model of Wharry et al. 41] uses partial diffusion coefficients defined by single effective diffusion barriers for each element-defect exchange fitted to a mean of migration barriers in a dilute configuration. This model does not exactly take into account the correlations between successive point defect jumps. It is nevertheless efficient for $\mathrm{Fe}-\mathrm{Cr}$ alloys, as diffusion properties are quite simple, due to the weak $\mathrm{Cr}-\mathrm{V}$ interactions. It would probably be less precise in case of drag effect.

Meanwhile, our model gives a coherent description of jump frequencies and transport coefficients in concentrated alloys and has the advantage that it can be also used to study radiation-accelerated precipitation (RAP) and radiationinduced precipitation (RIP). This model could however still be improved by correcting the overestimation of the negative coupling between $\mathrm{Cr}$ atoms and vacancy diffusion. It is difficult to fully reproduce the evolution of self-interstitial migration properties according to the local environment. Improved diffusion models with interactions at longer range and/or concentration dependent saddlepoint interactions would certainly help to reproduce these variations better.

Nevertheless we have confirmed qualitative conclusions of previous studies : the migration of vacancies toward sinks induces a depletion of $\mathrm{Cr}$ in the vicinity of sinks. The stability of mixed interstitials and their fast migration induces 
a tendency towards $\mathrm{Cr}$ enrichment at sinks. Measures of coupling coefficients and simulations of RIS have shown that, for concentrations and temperatures outside of the miscibility gap, the alloy tends to be enriched in $\mathrm{Cr}$ at sinks at temperatures lower than a threshold value and to be depleted in $\mathrm{Cr}$ at sinks at higher temperatures. The threshold temperature depends on the bulk concentration in a non-monotonic way.

We however note that the experimental observations in ferritic steels are far less simple 4 as already noticed in 9 . The main reason is probably that RIS results from a delicate balance between the enrichment due to self-interstitials and the depletion due to the vacancy in these ferritic alloys. The situation is therefore very different from the case of austenitic alloys where the elimination of vacancies and interstitials both contribute to a depletion of $\mathrm{Cr}$ and enrichment 520 of $\mathrm{Ni}$ [3, 44]. In ferritic steels, small variations in migration barriers can induce an inversion of tendencies [9. These variations can be in the order of the uncertainties in DFT calculations (see also Messina et al. [16]), making such multiscale approaches to RIS phenomena especially challenging.

Furthermore, any contribution affecting the migration barriers and the $L_{i j}$ coefficients could affect the segregation behavior:

- the influence of impurities, e.g. carbon, on the experimental profiles [7, 8] that are not included here. The carbon effect will be integrated in our model in future works.

- the influence of equilibrium segregation tendencies, that may reinforce or oppose the radiation induced ones, and will strongly depend on the interaction between $\mathrm{Cr}$ atoms and various point defects sinks.

- long range elastic interactions between sinks (e.g. dislocations) and solute atoms.

These contributions may be introduced in AKMC simulations and give fur535 ther insights into the mechanisms controlling RIS in ferritic steels.

The results presented in this paper may suggest that systematic experimental studies of RIS in pure binary Fe-Cr alloys (especially with minimum C content) depending on Cr content outside of the miscibility gap would help 
to better understand couplings between defects and chemical species evolutions

with $\mathrm{Cr}$ content. Moreover, most of RIS profiles have been observed at $1 \mathrm{dpa}$ and higher doses. To separate short term evolution (the segregation observed for a given density of point defect sinks), from later evolution due to microstructural changes, alloys could be analyzed at low doses (below 1 dpa, or even 0.1 dpa). By comparison with ion or neutron irradiations that lead to the creation

${ }_{545}$ of high densities of small dislocation loops in displacement cascades, electron irradiation would probably delay the evolution of the sink density. This may also help to separate the evolution at low and high doses.

\section{Acknowledgments}

We would like to thank E. Clouet for many fruitful discussions. This research has received partial funding from the Eurofusion IReMEV program and from the European Atomic Energy Community 7th Framework Program (FP7/20072011), under Grant agreement No. 212175 (GetMat project). This work also contributes to the Joint Program on Nuclear Materials (JPNM) of the European Energy Research Alliance (EERA). DFT calculations were performed using resources from DARI within project x2015096020. E. M. gratefully acknowledges the support of the US Department of Energy through the LANL/LDRD Program for this work.

\section{References}

[1] P. Yvon, F. Carré, Structural materials challenges for advanced reactor systems, Journal of Nuclear Materials 385:2 (2009) 217-222.

[2] S. Zinkle, J.T.Busby, Structural materials for fission and fusion energy, Materials Today 12:11 (2009) 12-19.

[3] M. Nastar, F. Soisson, Radiation-induced segregation, in: R. J. Konings (Ed.), Comprehensive Nuclear Materials, Elsevier, Oxford, 2012, pp. 471496. 
[4] Z. Lu, R. Faulkner, G. Was, B. Wirth, Irradiation-induced grain boundary chromium microchemistry in high alloy ferritic steels, Scripta Materialia 58 (10) (2008) $878-881$.

[5] G. S. Was, J. Wharry, B. Frisbie, B. D. Wirth, D. Morgan, J. Tucker, T. R. Allen, Assessment of radiation-induced segregation mechanisms in austenitic and ferritic-martensitic alloys, Journal of Nuclear Materials $411(1-3)(2011) 41-50$.

[6] J. Wharry, Z. Jiao, G. S. Was, Application of the inverse kirkendall model of radiation-induced segregation to ferritic-martensitic alloys, Journal of Nuclear Materials 425 (1-3) (2012) 117 - 124.

[7] R. Hu, G. D. Smith, E. Marquis, Effect of grain boundary orientation on radiation-induced segregation in a fe- 15.2 at.\% cr alloy, Acta Materialia 61 (9) (2013) $3490-3498$.

[8] E. Marquis, R. Hu, T. Rousseau, A systematic approach for the study of radiation-induced segregation/depletion at grain boundaries in steels, Journal of Nuclear Materials 413 (1) (2011) $1-4$.

[9] S. Choudhury, L. Barnard, J. Tucker, T. Allen, B. Wirth, M. Asta, D. Morgan, Ab-initio based modeling of diffusion in dilute bcc fe-ni and fe-cr alloys and implications for radiation induced segregation, Journal of Nuclear Materials 411 (1-3) (2011) $1-14$.

[10] L. Messina, M. Nastar, T. Garnier, C. Domain, P. Olsson, Exact ab initio transport coefficients in bcc Fe- $x(x=\mathrm{Cr}, \mathrm{Cu}, \mathrm{Mn}, \mathrm{Ni}, \mathrm{P}, \mathrm{Si})$ dilute alloys, Phys. Rev. B 90 (2014) 104203.

[11] D. Terentyev, P. Olsson, T. Klaver, L. Malerba, On the migration and trapping of single self-interstitial atoms in dilute and concentrated fecr alloys: Atomistic study and comparison with resistivity recovery experiments, Computational Materials Science 43 (4) (2008) 1183 - 1192. 
[12] V. Pechenkin, V. Molodtsov, V. Ryabov, D. Terentyev, On the radiationinduced segregation: Contribution of interstitial mechanism in fe-cr alloys, Journal of Nuclear Materials 433 (1-3) (2013) 372 - 377.

[13] H. Wiedersich, P. Okamoto, N. Lam, A theory of radiation-induced segregation in concentrated alloys, Journal of Nuclear Materials 83 (1) (1979) $98-108$.

[14] W. Wolfer, Drift forces on vacancies and interstitials in alloys with radiation-induced segregation, Journal of Nuclear Materials 114 (2-3) (1983) $292-304$.

[15] T. Garnier, N. Nastar, P. Bellon, D.-R. Trinkle, Solute drag by vacancies in body-centered cubic alloys, Phys. Rev. B 88 (2013) 134201.

[16] L. Messina, Z. Chang, P. Olsson, Ab initio modelling of vacancysolute dragging in dilute irradiated iron-based alloys, Nuclear Instruments and Methods in Physics Research Section B: Beam Interactions with Materials and Atoms 303 (0) (2013) $28-32$.

[17] E. Martinez, O. Senninger, C.-C. Fu, F. Soisson, Decomposition kinetics of fe-cr solid solutions during thermal aging, Phys. Rev. B 86 (2012) 224109.

[18] C. Pareige, F. Soisson, G. Martin, D. Blavette, Ordering and phase separation in ni-cr-al: Monte carlo simulations vs three-dimensional atom probe, Acta Materialia 47 (6) (1999) 1889 - 1899.

[19] C. Booth-Morrison, D. Seidman, D. Dunand, Effect of er additions on ambient and high-temperature strength of precipitation-strengthened al?zr?sc?si alloys, Acta Materialia 60 (8) (2012) 3643 - 3654.

[20] Z. Mao, C. Sudbrack, K. Yoon, G. Martin, D. Seidman, The mechanism of morphogenesis in a phase-separating concentrated multicomponent alloy, Nat Mater 6 (3) (2007) 210-216. 
[21] E. Clouet, L. Lae, T. Epicier, W. Lefebvre, M. Nastar, A. Deschamps, Complex precipitation pathways in multicomponent alloys, Nat Mater 5 (2006) 482-488.

[22] M. Levesque, E. Martinez, C.-C. Fu, M. Nastar, F. Soisson, A simple concentration-dependent pair interaction model for large-scale simulations of fe-cr alloys, Physical Review B 84 (2011) 184205.

[23] G. Bonny, D. Terentyev, L. Malerba, New contribution to the thermodynamics of fe-cr alloys as base for ferritic steels, Journal of Phase Equilibria and Diffusion 31-5 (2010) 439-444.

[24] W. Xiong, M. Selleby, Q. Chen, J. Odqvist, Y. Du, Phase equilibria and thermodynamic properties in the fe-cr system, Critical Reviews in Solid State and Materials Sciences 35 (2) (2010) 125-152.

[25] C.-C. Fu, F. Willaime, P. Ordejon, Stability and mobility of mono- and di-interstitials in alpha-fe, Phys. Rev. Lett. 92 (2004) 175503.

[26] P. Olsson, Ab initio study of interstitial migration in fecr alloys, Journal of Nuclear Materials 386-388 (2009) 86 - 89.

[27] A. D. LeClaire, Correlation effects in diffusion in solids, Academic, New York, 1970, Ch. 10, p. 261.

[28] O. Senninger, E. Martnez, F. Soisson, M. Nastar, Y. Brchet, Atomistic simulations of the decomposition kinetics in fecr alloys: Influence of magnetism, Acta Materialia 73 (0) (2014) 97 - 106.

[29] P. Olsson, C. Domain, J. Wallenius, Ab initio study of cr interactions with point defects in bcc fe, Phys. Rev. B 75 (2007) 014110.

[30] P. Giannozzi, S. Baroni, N. Bonini, M. Calandra, R. Car, C. Cavazzoni, D. Ceresoli, G. L. Chiarotti, M. Cococcioni, I. Dabo, Quantum espresso: a modular and open-source software project for quantum simulations of materials, J. Phys.: Condens. Matter 21 (2009) 395502. 
[31] C. Domain, C. S. Becquart, Ab initio calculations of defects in fe and dilute fe-cu alloys, Phys. Rev. B 65 (2001) 024103.

[32] T. P. C. Klaver, R. Drautz, M. Finnis, Magnetism and thermodynamics of defect-free fe-cr alloys, Physical Review B 74 (2006) 094435.

[33] A. Bortz, M. Kalos, J. Lebowitz, New algorithm for Monte-Carlo simulation of ising spin systems, Journal of computational physics 17 (1) (1975) 10-18.

[34] A. R. Allnatt, A. D. Lidiard, Atomic transport in solids, Cambridge University Press, 1993.

[35] F. Soisson, Kinetic monte carlo simulations of radiation induced segregation and precipitation, Journal of Nuclear Materials 349 (3) (2006) 235 - 250.

[36] F. Soisson, C.-C. Fu, Atomistic simulations of copper precipitation and radiation induced segregation in -iron, Solid State Phenomena 139 (2008) 107.

[37] W. Wagner, L. Rehn, H. Wiedersich, V. Naundorf, Radiation-induced segregation in ni-cu alloys, Physical Review B 28 (12) (1983) 6780.

[38] R. Sizmann, The effect of radiation upon diffusion in metals, Journal of Nuclear Materials 6970 (0) (1978) 386 - 412.

[39] Y. Grandjean, P. Bellon, G. Martin, Kinetic model for equilibrium and nonequilibrium segregation in concentrated alloys under irradiation, Physical Review B 50 (6) (1994) 4228.

[40] L. Rehn, P. Okamoto, H. Wiedersich, Dose dependence of radiation-induced segregation in ni-1 at\% si, Journal of Nuclear Materials 80 (1) (1979) 172179.

[41] J. P. Wharry, G. S. Was, The mechanism of radiation-induced segregation in ferriticmartensitic alloys, Acta Materialia 65 (0) (2014) 42 - 55. 
[42] V. Kuksenko, C. Pareige, P. Pareige, Cr precipitation in neutron irradiated industrial purity fecr model alloys, Journal of Nuclear Materials 432 (13) (2013) $160-165$.

[43] M. Nastar, P. Bellon, G. Martin, J. Ruste, Role of interstitial and interstitial-impurity interaction on irradiation-induced segregation in austenitic steels, in: Symposium P Science and Technology of Semiconductor Surface Preparation, Vol. 481 of MRS Proceedings, 1997.

[44] M. Nastar, A mean field theory for diffusion in a dilute multi-component alloy: a new model for the effect of solutes on self-diffusion, Philosophical Magazine 85 (32) (2005) 3767-3794.

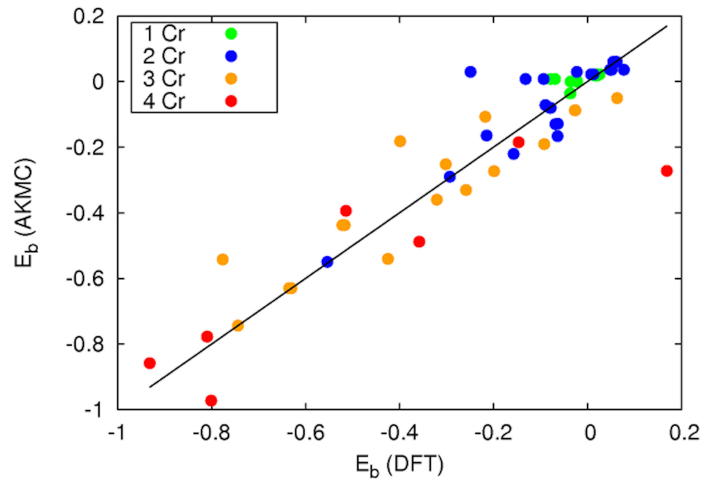

Figure 1: Binding energies between one $\langle 110\rangle$ dumbbell and $\mathrm{Cr}$ atoms in Fe. Comparison between the AKMC model and the DFT calculations. 


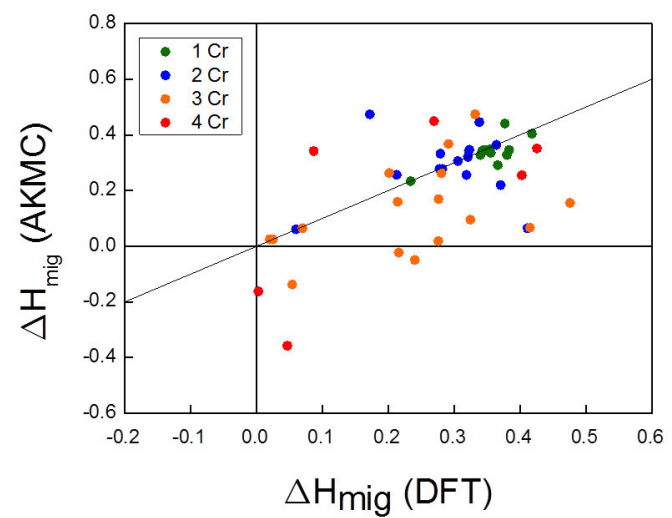

Figure 2: Migration energies of a $\langle 110\rangle$ dumbbell, with up to four $\mathrm{Cr}$ neighbors in $\mathrm{Fe}$. Comparison between the AKMC model and the DFT calculations.

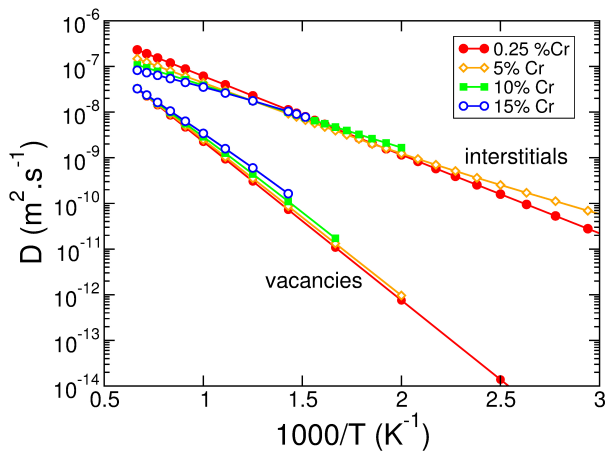

Figure 3: Vacancy and interstitial tracer diffusion coefficients in $\mathrm{Fe}-\mathrm{Cr}$ alloys at various nominal compositions. AKMC simulations $10^{5} \times 10^{5}$ MCS in stable and metastable solutions 


\begin{tabular}{|c|c|c|c|c|}
\hline SIA configuration & $\begin{array}{l}\text { DFT-PAW } \\
\text { (this study) }\end{array}$ & DFT-PAVW & 2BM-E: $P^{b}$ & $\begin{array}{l}\text { AKMC } \\
\text { (this study) }\end{array}$ \\
\hline \multicolumn{5}{|c|}{$<110>$ dumbbells in $\mathrm{Fe}$} \\
\hline 1 & +0.022 & +0.024 & +0.14 & +0.022 \\
\hline 2 & -0.038 & -0.065 & +0.16 & -0.036 \\
\hline 3 & +0.016 & +0.05 & +0.11 & +0.018 \\
\hline 4 & +0.078 & +0.154 & -0.19 & +0.012 \\
\hline 5 & -0.063 & -0.041 & -0.05 & -0.166 \\
\hline 6 & +0.048 & & & +0.036 \\
\hline 7 & -0.078 & -0.023 & +0.13 & -0.080 \\
\hline 8 & -0.293 & -0.209 & -0.07 & -0.290 \\
\hline 9 & +0.055 & +0.154 & +0.04 & +0.060 \\
\hline 10 & -0.612 & & & -0.713 \\
\hline 11 & -0.554 & -0.425 & -0.29 & -0.550 \\
\hline 12 & -0.744 & & & -0.744 \\
\hline \multicolumn{5}{|c|}{$<110>$ dumbbells in $\mathrm{Cr}$ (NM) } \\
\hline 13 & +0.920 & & & +1.002 \\
\hline 14 & +2.106 & & & +1.164 \\
\hline \multicolumn{5}{|c|}{$<111>$ dumbbells in $\mathrm{Fe}$} \\
\hline 15 & +0.353 & +0.373 & +0.40 & \\
\hline 16 & +0.207 & +0.223 & -0.02 & \\
\hline
\end{tabular}

Table 2: Binding energies of various configurations obtained in our DFT calculations and AKMC model. $\mathrm{Cr}$ atoms are in a dark color and $\mathrm{Fe}$ atoms are in white. Values in $\mathrm{eV}$ 


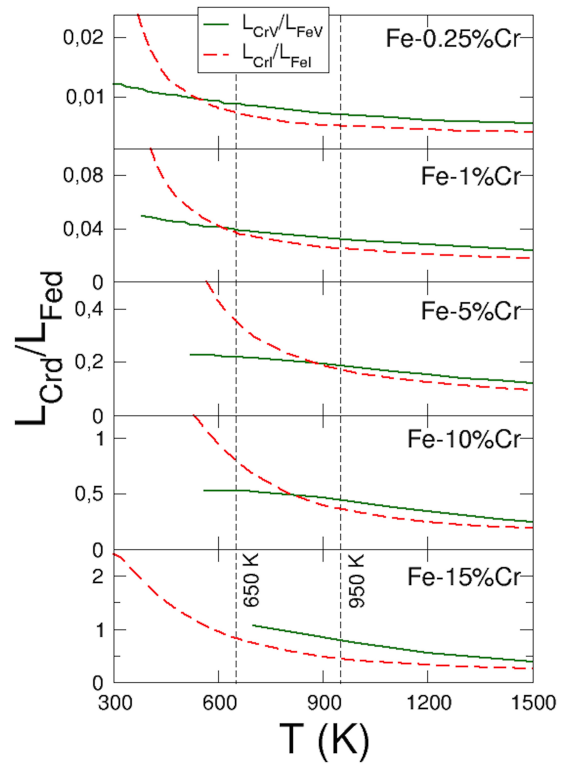

Figure 4: evolution of $L_{C r I} / L_{F e I}$ and $L_{C r V} / L_{F e V}$ with $\mathrm{T}$ for different alloy compositions. AKMC measurements: $10^{5} \times 10^{5} \mathrm{MCS}$, in stable and metastable solid solutions.
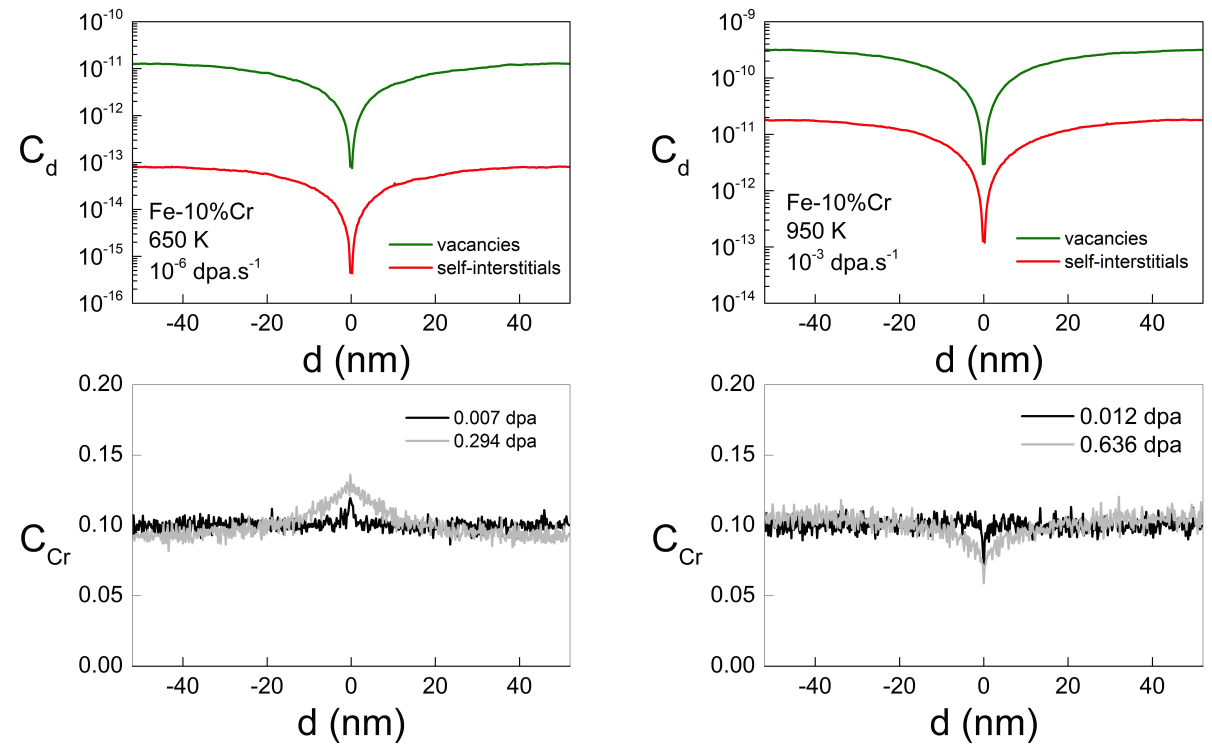

Figure 5: Steady-State point defects and Cr concentration profiles in the vicinity of a perfect planar sink for an alloy at $10 \% \mathrm{Cr}$ left) at $650 \mathrm{~K}$ irradiated by a flux of $10^{-6} \mathrm{dpa}_{\mathrm{s}}{ }^{-1}$ (at 0.007 and $0.294 \mathrm{dpa}$ ) right) at $950 \mathrm{~K}$ irradiated by a flux of $10^{-3} \mathrm{dpa}_{\mathrm{s}} \mathrm{s}^{-1}$ (at 0.012 and $0.636 \mathrm{dpa}$ ). 


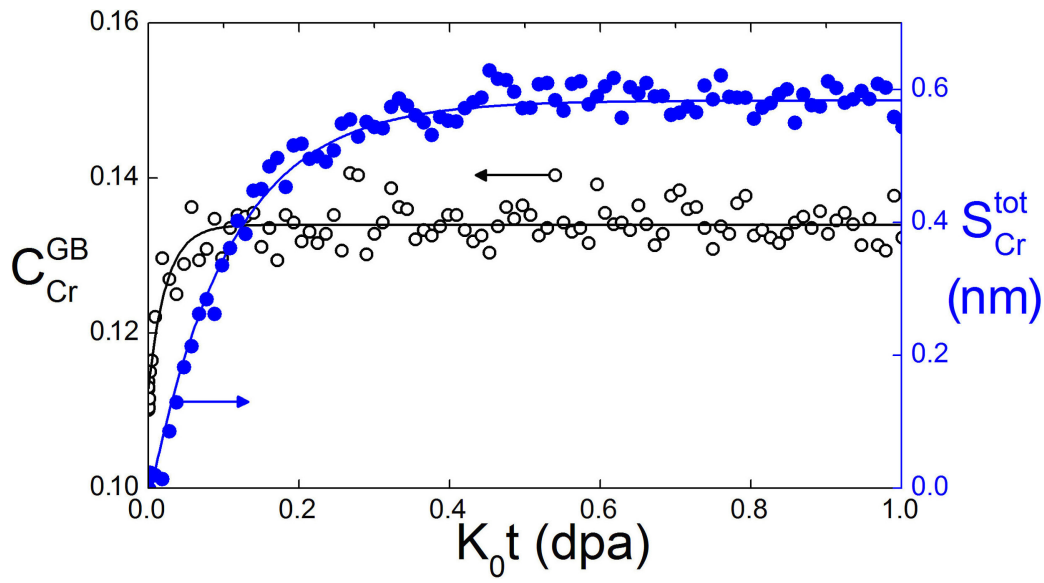

Figure 6: Concentration of $\mathrm{Cr}$ at $\operatorname{sink}\left(C_{C r}^{G B}\right)$ and total quantity of $\mathrm{Cr}$ segregated $\left(S_{C r}^{t o t}\right)$ as a function of dose. Simulations for an alloy at $10 \% \mathrm{Cr}$ and $650 \mathrm{~K}$ using a simulation box of $256 \times 64 \times 64$ bcc sites.
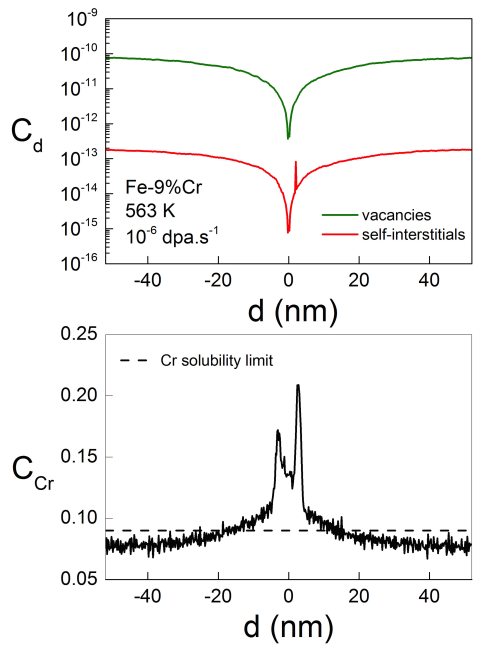

Figure 7: AKMC simulations of RIS and RIP near the grain boundary in a Fe-9\%Cr alloy at $563 \mathrm{~K}$ under a dose rate of $10^{-6} \mathrm{dpa}_{\mathrm{s}} \mathrm{s}^{-1}$ and a dose of $0.200 \mathrm{dpa}$. (left) point defects and $\mathrm{Cr}$ concentration profiles and (right) map of $\mathrm{Cr}$ atoms in the simulation box 

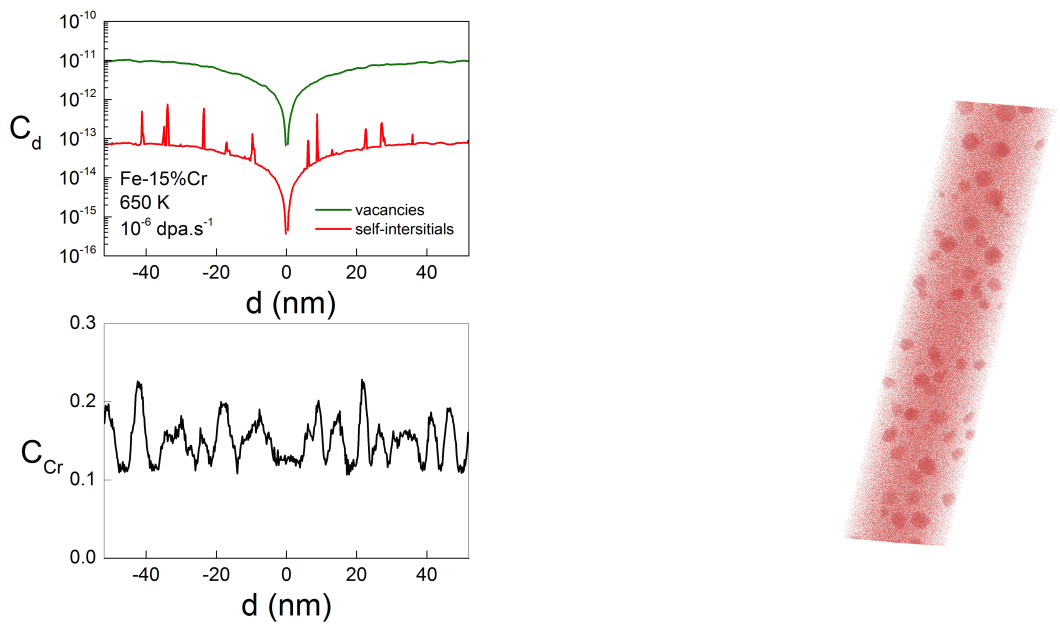

Figure 8: AKMC simulation of RIS near a grain boundary in a $\mathrm{Fe}-15 \% \mathrm{Cr}$ alloy at $650 \mathrm{~K}$ under a dose rate of $10^{-6} \mathrm{dpa}^{-1}$. (left) Steady-State point defects concentration profiles and (right) Atomic configuration at $0.136 \mathrm{dpa}$ (only the $\mathrm{Cr}$ atoms are shown). 